\title{
DIAGNÓSTICO DE LOS NIVELES DE MADUREZ DE LA GESTIÓN DEL PROCESO DE DESARROLLO DE PRODUCTOS EN INDUSTRIAS ALIMENTICIAS
}

Leticia Milena Arcusin (larcusin@fiq.unl.edu.ar) - Departamento de Ingeniería y Gestión Industrial, Facultad de Ingeniería Química, Universidad Nacional del Litoral

Melisa De Greef (melisadegreef@gmail.com) - Departamento de Ingeniería y Gestión Industrial, Facultad de Ingeniería Química, Universidad Nacional del Litoral

Germán Rossetti (groseti@fiq.unl.edu.ar) - Departamento de Ingeniería y Gestión Industrial, Facultad de Ingeniería Química, Universidad Nacional del Litoral

Daniela Ferreira Campos (ferreiracamposdaniela@gmail.com) - Departamento de Ingeniería y Gestión Industrial, Facultad de Ingeniería Química, Universidad Nacional del Litoral

\section{RESUMEN}

El desarrollo de nuevos productos es una actividad esencial para la supervivencia y competitividad de las empresas. En el caso de las industrias productoras de alimentos, la Gestión del Proceso de Desarrollo de Productos (PDP) reviste una importancia crucial, debido al escenario competitivo en el que se desenvuelven. Sin embargo, en estas empresas se observan distintos grados de sistematización del proceso. En este sentido, el Nivel de Madurez brinda pautas sobre el grado en que las mejores prácticas se institucionalizan y se hacen efectivas en toda la organización. En la literatura seleccionada se reconocen cinco Niveles de Madurez: Básico, Intermedio, Medible, Controlado y Mejora Continua. En el presente trabajo se realiza el diagnóstico del nivel de madurez de la Gestión del Proceso de Desarrollo de Productos (PDP) en empresas productoras de alimentos del Gran Santa $\mathrm{Fe}$ (Argentina). Se seleccionan once empresas y se lleva a cabo una investigación exploratoria mediante entrevistas y observación in situ. Entre las principales conclusiones, se determina que las empresas entrevistadas poseen Niveles de Madurez Básico e Intermedio. Particularmente, se observan notorias diferencias en relación al tamaño de las empresas (a mayor tamaño, mayor grado de sistematización del proceso del PDP y mayor nivel de madurez).

Palabras clave: Niveles de Madurez, Desarrollo de Productos, Industrias alimenticias.

Área: Melhoria do processo de desenvolvimento de produtos e serviços

\section{INTRODUCCIÓN}

El proceso de desarrollo de productos (en adelante, PDP) es la tarea sistemática que tiene como propósito generar nuevos productos, ya sea introduciendo modificaciones en un producto existente o generando otro completamente nuevo y original (LERMA KIRCHNER, 2010). En el caso de las industrias productoras de alimentos, el PDP reviste una importancia crucial, debido a que el escenario donde se desarrollan las empresas del sector se caracteriza por altos niveles de competitividad y una evolución en diversas perspectivas: seguridad alimentaria, sustentabilidad, embalaje, entre otras (ABU, 2012).

Las empresas que han logrado sistematizar la Gestión de su PDP, en general grandes organizaciones, se ven ampliamente beneficiadas en el desarrollo de sus negocios. No 
obstante, en muchas organizaciones, la Gestión del PDP no se encuentra documentada ni estructurada. Se realizan una serie de actividades, con distinto grado de formalización, sin llegar a ser un proceso integral y sistematizado. El grado de desarrollo de este tipo de prácticas es un indicador del nivel de madurez de las empresas.

La utilización de ese concepto hace posible diagnosticar el patrón actual de determinado proceso de negocio; y, además, orientar a expertos, departamentos o incluso unidades de negocio sobre lo que se puede hacer en términos de gestión para que ese proceso alcance estándares de desempeño superior al actual.

El objetivo del presente trabajo es realizar un estudio sobre la Gestión del Proceso de Desarrollo de Productos en empresas productoras de alimentos de la Provincia de Santa $\mathrm{Fe}$ para determinar su nivel de madurez. La investigación se enmarca en un Proyecto orientado a proponer un Modelo de Gestión del PDP para empresas productoras de alimentos de la Provincia de Santa Fe que toma como referencia el Modelo propuesto por Rozenfeld et al (2006). El trabajo presenta los resultados preliminares correspondientes a once empresas de la Región 3 Nodo Santa Fe, según regionalización definida por el Estado Provincial a partir del año 2008.

\section{METODOLOGIA}

La presente investigación es de carácter exploratorio-descriptivo (SAUTU et al, 2005), y tiene como fin principal realizar un estudio del Nivel de Madurez de la Gestión del Proceso de Desarrollo de Productos en empresas productoras de alimentos radicadas en la provincia de Santa Fe (Argentina), pertenecientes a tres sectores de actividad, seleccionados en función de su importancia para la región y definidos en base a la clasificación propuesta para la Industria Manufacturera por un organismo público nacional: lácteos, insumos y carnes. Para el análisis, se describen las prácticas habituales de las empresas bajo estudio y se procede a compararlas con las actividades propuestas por un Modelo de Referencia para la industria de alimentos.

Con el objetivo de conocer con profundidad la forma en que las empresas efectúan la Gestión del PDP, se realiza un relevamiento a través de entrevistas semi-estructuradas y observación in situ en once empresas, denominadas en adelante empresas A, B, C, D (sector lácteo), E, F, $\mathrm{G}, \mathrm{H}$ (sector insumos), I, J y K (sector carnes). Las entrevistas fueron realizadas al personal vinculado con el desarrollo de productos (empresas A, B, C, E y F) y a la gerencia (empresas D, G, H, I, J y K).

Las entrevistas fueron analizadas mediante matrices organizadas a partir de las macrofases (Pre-Desarrollo, Desarrollo y Pos-Desarrollo) reconociendo las actividades que cada empresa realiza (o no) en función de las propuestas por el modelo. Para la observación se utilizaron fichas de registro donde se evaluaron aspectos como: tamaño y estado de las instalaciones, tecnología existente, nivel jerárquico del entrevistado, entre otros.

\section{MARCO TEORICO}

La Gestión del PDP implica la elección de una metodología de trabajo que comienza por la identificación de una necesidad insatisfecha en la sociedad hasta llegar a obtener un producto que la satisfaga de la mejor manera posible (ARCUSIN; ROSSETTI, 2014). En el sector alimenticio, la gestión de este proceso es crucial, dada la creciente exigencia de los consumidores, la saturación de productos en un mercado maduro y los constantes cambios que introducen las firmas líderes. 
Sin embargo, muchas de ellas realizan una serie de actividades que cuentan con distinto grado de formalización, pero no llegan a ser un proceso integral formalizado (DE GREEF et al, 2014). Ello varía en función del nivel de madurez de las empresas, el cual depende de aspectos contextuales y estructurales de las organizaciones, y, en relación a la gestión del PDP, indica en qué medida las mismas aplican mejores prácticas en tal proceso (AGUIRRE MAYORGA y CÓRDOBA PINZÓN, 2008). El estudio de los Niveles de Madurez permite diagnosticar el proceso de la empresa (nivel actual de madurez) y guiar a los expertos sobre formas de intervención, en términos de gestión, para alcanzar un rendimiento superior (PENSO, 2003).

En base a Chrissis (2003), Rozenfeld et al. (2006) propone cinco niveles de madurez; y cada uno incorpora nuevas actividades a las del nivel anterior. Los niveles Básico e Intermedio se dividen a su vez en cuatro subniveles.

1. Básico: se realizan sólo algunas actividades esenciales del PDP:

1.1: Contiene algunas actividades relativas al área "Ingeniería de Producto". Los requisitos son definidos de forma no sistemática. Se realiza un esbozo del producto en relación a esas características (nivel básico de concepción del producto). La fase de preparación de la producción comprende solo la adquisición de los recursos necesarios para fabricar el producto, adquisición que tampoco se realiza de forma sistemática;

1.2: Se realizan actividades relacionadas con las áreas "Marketing" y "Calidad". Existe una integración inicial entre la planificación estratégica de la empresa y la estrategia de producto, aunque es informal y por medio del diálogo, teniendo en cuenta la experiencia de los gerentes y responsables. Para la definición de los requisitos del producto, se realiza una evaluación informal del ciclo de vida del producto: se percibe que existen varios interesados en el producto, no sólo los clientes. Comienzan a realizarse algunas actividades de lanzamiento del producto y otras relativas a atender la legislación vigente. Además, se llevan a cabo iniciativas para mejorar el PDP a partir de proyectos de transformación simples, sin un abordaje sistemático.

1.3: Son realizadas actividades específicas relacionadas con las áreas "Ingeniería de Procesos", "Producción" y "Suministros". La empresa realiza una planificación del proceso de forma sistemática y además cronograma sus acuerdos con los principales proveedores;

1.4: Se realizan las actividades relacionadas con "Gestión de Proyectos" y "Costos". La empresa piensa en un portfolio de productos, analizando cada proyecto de forma relativa. La planificación del proyecto incluye el análisis de la viabilidad económica y el uso de sistemas de gerenciamiento de proyectos. La empresa comienza a aplicar los conceptos de aprobación de fase (gate). La planificación del lanzamiento es más elaborada, integrando las campañas de marketing al PDP;

2. Intermedio: las actividades son realizadas bajo patrones y sus resultados son predecibles. Se utilizan además métodos y herramientas probadas de desarrollo de productos:

2.1: La empresa empieza a definir familias de productos en el estudio del porfolio. La planificación del proyecto incluye un análisis de riesgos y procedimientos de calidad. La mayor diferencia con niveles anteriores se encuentra en la fase de desarrollo (área "Ingeniería de Producto"), donde se realiza un modelado funcional y surgen los principios de solución, que son agregados a las concepciones alternativas. Luego del lanzamiento del producto, las actividades orientadas a realizar el seguimiento en el mercado son formalmente realizadas y se designa un equipo de acompañamiento de producto. El gerenciamiento de los cambios se da de manera informal, no sistemática; 2.2: Son realizadas actividades relacionadas con las áreas "Marketing" y de "Calidad". 
La gestión del portfolio se realiza en forma integrada con la planificación estratégica de la empresa;

2.3: Adiciona actividades relacionadas a las áreas de "Ingeniería de Proceso", "Producción" y "Suministros". Se produce la integración con los miembros de la cadena de suministros desde las primeras fases del desarrollo;

2.4: Se realizan actividades relacionadas a las áreas de "Gestión de Proyectos", "Costos" y "Medio Ambiente". Todas las actividades de la Gestión de Proyectos son realizadas y se implementan gates en todas las fases. El seguimiento de costos, cantidades y precios pronosticados son monitoreados constantemente, como también los riesgos del proyecto. El desarrollo sustentable (planes de reutilización, reciclaje y descarte) es considerado cuando se define el plan de fin de vida del producto;

3. Medible: existen y se utilizan indicadores de desempeño para todas las actividades, así como indicadores de gestión del proyecto. Si bien la empresa utiliza la medición, ello no constituye un procedimiento definido. Las acciones de corrección ocurren de forma no sistemática;

4. Controlado: se lleva a cabo el control de todas las actividades, en base a los indicadores del nivel anterior (medición y control). La empresa trabaja de forma sistemática para corregir actividades;

5. Mejora continua: en todas las instancias se aplica la mejora continua (medición, control y mejora continua), desde lo referente al producto y al proceso de fabricación hasta lo concerniente a la transformación del propio PDP. En este momento, todas las prácticas del PDP son aplicadas.

\section{RESULTADOS Y DISCUSIÓN}

\subsection{Caracterización de las empresas}

Las empresas analizadas están localizadas en la Región 3 Nodo Santa Fe, según regionalización del Estado Provincial.

En relación al tamaño de las organizaciones, según la clasificación de la Secretaría de Emprendedores y de la Pequeña y Mediana Empresa de Argentina, las empresas A, B, C, D, E, H, se clasifican entonces como Grandes Empresas, I, K y L como Medianas, mientras que F, G, J y M como Pequeñas.

Las empresas A, B, C y D pertenecen al sector lácteo y ofrecen diferentes líneas de productos (la empresa A cuenta con 207 productos, B con 59, C con 160 y la empresa D con 20). Las actividades de comercialización de $\mathrm{D}$ se definen dentro del país, mientras que $\mathrm{A}, \mathrm{B}$ y $\mathrm{C}$ cubren el territorio nacional e internacional.

Las empresas E, F, G y H pertenecen al sector de insumos destinados a diversas industrias: láctea, golosinas, bebidas, panificación, carnes, etc., e incluso pueden abarcar otras industrias como cosmética, fotografía, entre otras. La empresa $\mathrm{E}$ se dedica a la producción de preparados de fruta, la empresa $\mathrm{F}$ a proveer soluciones basadas en gelatinas e hidrolizados, $\mathrm{G}$ a la elaboración de conservas de tomate y fruta abrillantada, y $\mathrm{H}$ a la producción de deshidratados y congelados de origen vegetal.

Las empresas I, J y K pertenecen al sector de carnes. I elabora fiambres, embutidos y cortes de cerdo para la venta al público y abastece a zonas regionales. Las actividades de la empresa $\mathbf{J}$ se basan en la cría porcina de genética, y sus productos son comercializados mediante franquicias localizadas en la ciudad de Santa Fe. La empresa K se dedica a la elaboración y envasado de diferentes productos derivados del pollo. 


\subsection{Análisis del PDP por Sectores de actividad}

\subsubsection{Sector Lácteo}

Todas las empresas analizadas realizan actividades vinculadas a la Ingeniería de Producto, que son responsabilidad de personal de desarrollo o área encargada del desarrollo (según la empresa recibe diferentes denominaciones: Departamento de Desarrollo, Departamento de I+D y Departamento de Calidad). Existen diferencias entre las empresas en lo que respecta a la cantidad de tareas y a la profundidad de las mismas. En la empresa B el proceso se orienta a lograr muestras piloto satisfactorias por medio de "prueba y error"; en las empresas A y C evidencia un mayor grado de detalle en tareas vinculadas con el diseño informacional, diseño conceptual y diseño detallado; mientras que en D no existe un área específica encargada de desarrollo.

En relación a la Calidad, las empresas ponen de relieve la adecuada ejecución de Análisis Fisicoquímicos y Microbiológicos y de Determinación de Vida Útil mediante métodos específicos, como así también de Análisis Sensoriales con el objeto de lograr la aceptación del consumidor (sensibilidad, apariencia, color, sabor, textura, dimensiones y usos). Las empresas A, B y C realizan internamente estos análisis, mientras que la empresa I encarga parte de los estudios a laboratorios externos y no cuenta con un personal especializado para los Análisis Sensoriales. Otro aspecto que A, B y C consideran indispensable para garantizar la calidad es la relación con los proveedores, por ello existe una integración en la cadena de suministros.

En cuanto al planeamiento estratégico, a excepción de $\mathrm{D}$, las empresas reconocen la importancia de alinear la planificación del PDP con la planificación estratégica de la empresa, analizando familias de productos según el portfolio de la empresa, actividades en las que el área de Marketing tiene especial relevancia. Por ello, la mayoría de las veces, los lanzamientos o re-lanzamientos de productos se corresponden con decisiones estratégicas relacionadas a posicionar productos, ampliar mercados, proteger posiciones de liderazgo, entre otras.

En relación a la aprobación de las diferentes fases, existen diferencias entre las empresas. A, B y C tienen mecanismos estructurados de comunicación y de instancias de aprobación (hitos o "gates"). En la empresa C, que aplica todas las actividades de la Gestión de Proyectos, no es posible avanzar en el desarrollo de un proyecto si no se aprueban una serie de gates que se traducen en informes ("Ficha de Especificaciones del Producto", "Ficha de Proceso y Formulación", "Ficha de Proveedores", entre otras). En la empresa A, la aprobación de cada paso a realizarse en el desarrollo se asienta en de forma unificada en una Ficha de Producto, que reúne las especificaciones de cada proyecto, resulte o no en un lanzamiento efectivo de producto. En B no existe un mecanismo formal de aprobación, no obstante se siguen las mismas prácticas en cada desarrollo. En D no existen pasos establecidos.

Para el lanzamiento del producto las empresas A, B y C se enfocan en la definición de estrategias comerciales, en relación a precio, canales de distribución y promoción. En D se siguen las prácticas tradicionales de la empresa, excepto que el producto requiera algún tipo de promoción o distribución específica.

Luego del lanzamiento, las empresas A, B y C realizan el seguimiento de los productos en el mercado, comparando las proyecciones elaboradas con los resultados obtenidos, y, en base a esta información, se decide la continuidad o no del producto. D no efectúa un seguimiento formal, siendo fundamentalmente las situaciones de quejas o devoluciones de producto las que obligan a la empresa a revisar sus estrategias. 


\subsubsection{Sector Insumos}

En este sector existen notorias diferencias en las empresas entrevistadas, en lo que respecta a la Ingeniería de Producto y al PDP en general.

Un grupo de empresas, E y F, muestra un alto grado de estandarización en la Gestión del PDP. Los potenciales desarrollos son guiados por el Departamento I+D, encargado de monitorear el avance de PDP.

En E, se genera un Brief (expediente) que contiene datos específicos del proyecto (objetivos, formulación, consumidores, fechas, ventas, costos) y el documento Análisis de riesgo, donde se evalúa el impacto potencial del proyecto. Todos los desarrollos son conducidos por un mismo equipo de trabajo, conformado por integrantes de diferentes áreas.

La empresa $\mathrm{F}$ posee diferentes mecanismos de gestión del PDP según el tipo de proyecto (PMO o Workrequest): en los PMO (proyectos de gran magnitud, innovadores para la empresa), se realiza el Project Proposal (Propuesta de Proyecto), donde se define la idea, objetivos, alcance, estudio de costos y beneficios, requisitos, informaciones de proyectos anteriores y plan general de hitos; también se detallan los potenciales riesgos sobre Seguridad Alimentaria, Salud y Seguridad ocupacional y Aspectos Ambientales. De acuerdo a las características del proyecto, se selecciona un Project Manager (Líder) quien guía al equipo de trabajo, diferente para cada proyecto. Para los Workrequest (desarrollos de menor magnitud, menor innovación, y rápida ejecución), se realiza un Workplan (Plan de Trabajo), y las tareas se simplifican.

En ambas empresas, el PDP continúa guiado por el Departamento de Investigación y Desarrollo en las áreas de laboratorio, donde se efectúan muestras y comienza una serie de loops (retroalimentaciones) con el cliente hasta lograr resultados satisfactorios. Luego de una serie de escalamientos industriales, se incorpora el insumo a la planificación de la producción de la empresa. Uno de los puntos críticos para las empresas es la Calidad, por ello, especialmente para las pruebas de laboratorio, se elaboran Planes de trabajo que contienen información detallada de las formulaciones esperadas, análisis a realizar y cronogramas.

En un segundo grupo de empresas, $\mathrm{G}$ y $\mathrm{H}$, el proceso de desarrollo no sigue un lineamiento definido, sino que se limita a lograr una formulación adecuada del producto. Si bien la Calidad se reconoce importante, la mayoría de los desarrollos requieren la contratación de laboratorios externos y, para el Análisis Sensorial, no se cuenta con paneles especializados.

El primer grupo de empresas reconoce la importancia de alinear la planificación del PDP con la planificación estratégica de la empresa: muchos de los productos se corresponden a solicitudes de clientes, y por ello las empresas consideran necesario dar respuesta en la medida en que guarden relación con las estrategias corporativas, aspecto en que juega un rol central el área de Marketing. En el caso de G y H, el desarrollo de productos es esporádico, y responde a oportunidades del entorno más que a decisiones estratégicas de la empresa.

En cuanto a la aprobación de fases, existen notorias diferencias entre los grupos de empresas. En la empresa $\mathrm{F}$ la no es posible avanzar en el desarrollo de un proyecto si no se aprueban determinados hitos, que se reflejan en informes particulares (Project Charter, Project Development and Transfer, entre otros). En la empresa E la aprobación de cada paso a realizarse en el desarrollo se centraliza en una Hoja de proceso, que reúne las especificaciones de cada proyecto. En $\mathrm{H}$ no existe un mecanismo formal de aprobación, no obstante se siguen las mismas prácticas en cada desarrollo. En G no existen pasos establecidos.

Finalmente, en lo que refiere al lanzamiento del producto, se observa un bajo grado de sistematización. En E y F puede deberse, por un lado, a que la mayor parte de los productos 
surgen a partir de pedidos de clientes y, por otro, a que las empresas no se vinculan con consumidores finales y por lo tanto posicionar sus productos no representa una actividad esencial. En G y H la estrategia se basa en comenzar a comercializar el producto en puntos de ventas con los que existe relación de cercanía para evaluar la aceptación de los clientes.

Una vez efectuado el lanzamiento, en $\mathrm{E}$ y $\mathrm{F}$ el seguimiento del producto, realizado por el departamento de Marketing, se basa en la comparación de las proyecciones elaboradas con los resultados obtenidos, y las actividades de esta fase se orientan fundamentalmente al Retiro de productos en caso de que ocurra algún incidente. En la empresa $\mathrm{H}$ el seguimiento se realiza desde el área de Marketing, a partir de la opinión de los clientes y de la comparación de los pronósticos de venta y los resultados obtenidos, información con la que se decide la continuidad, modificación o retiro del producto. En G existe únicamente existe un Registro de no conformidad del cliente.

\subsubsection{Sector Carnes}

El sector presenta baja sistematización en cuanto a las áreas Ingeniería de Producto, especialmente en el caso de I, que lleva a cabo el desarrollo de manera menos estructurada: una vez que se selecciona la idea de producto, no se sigue una metodología específica, sino que se intenta materializar el producto a partir de "prueba y error". En las empresas J y K, el proceso de desarrollo del producto no sigue un lineamiento definido; no obstante es llevado a cabo por un equipo donde participan el encargado de planta y responsables de ventas y administración, y se realizan reuniones periódicas para revisar el avance del proyecto en lo que respecta al producto y procesos.

En relación a la Calidad, J y K realizan una serie de pruebas y testeos internamente, y en caso de desarrollos específicos se contrata un laboratorio externo; y, para el Análisis Sensorial, no se cuenta con paneles especializados aunque es realizado por el mismo personal en cada proyecto. La relación con los proveedores resulta fundamental para lograr la continuidad en las características de los productos ofrecidos, por ello se planifica en forma sistemática los requerimientos de producto y los cronogramas de entrega. En el caso de I, la mayoría de los desarrollos requieren la contratación de laboratorios externos, el Análisis Sensorial es informal y no existe planificación con los proveedores.

En relación al lanzamiento del producto, la estrategia en I y J consiste en comenzar con su incorporación en algunos puntos de venta para evaluar el impacto en los clientes; mientras que en $\mathrm{K}$ se trabaja con determinados distribuidores para evaluar la aceptación del producto en el consumidor final.

Las tres empresas manifiestan no contar con mecanismos formales de planificación estratégica ni de aprobación de fases.

En J y K el seguimiento de los productos se realiza de manera cualitativa (opinión del cliente) como también de manera cuantitativa (nivel de ventas y rentabilidad), y con esa información se toma la decisión de retirar el producto del mercado u ofrecerlo de forma diferente; el área de Marketing juega un rol indispensable en estas actividades. En el caso de I, únicamente se analiza si las ventas del producto generan ganancias y en ese caso se continúa su producción.

A modo de síntesis, la Figura 1 muestra los Niveles de Madurez según las mejores prácticas de las empresas analizadas. 
Figura 1. Niveles de Madurez de las empresas. Elaboración propia en base a Rozenfeld et al. (2006)

\begin{tabular}{|c|c|c|c|c|c|c|c|c|c|c|c|}
\hline \multicolumn{12}{|l|}{ NIVELES DE MADUREZ } \\
\hline \multicolumn{12}{|l|}{ 5. Mejora Continua } \\
\hline \multicolumn{12}{|l|}{ 4. Controlado: Existen controles y correcciones } \\
\hline \multicolumn{12}{|l|}{ 3. Medible: Los resultados son medibles } \\
\hline 2.4 Gestión de Proyectos, Costos y Medio Ambiente & & & $\mathbf{C}$ & & $\mathbf{E}$ & $\mathbf{F}$ & & & & & \\
\hline 2.3 Ingeniería de Procesos, Producción y Suministros & $\mathbf{A}$ & B & & & & & & & & & \\
\hline \multicolumn{12}{|l|}{ 2.2 Marketing y Calidad } \\
\hline \multicolumn{12}{|l|}{2.1 Ingeniería de Producto } \\
\hline \multicolumn{12}{|l|}{$\begin{array}{l}\text { 2. Intermedio: utiliza patrones y métodos probados; } \\
\text { gestiona actividades; es repetitivo }\end{array}$} \\
\hline \multicolumn{12}{|l|}{ 1.4 Gestión de Proyectos y Costos } \\
\hline 1.3 Ingeniería de Procesos, Producción y Suministros & & & & & & & & $\mathbf{H}$ & & $\mathbf{J}$ & $\mathbf{K}$ \\
\hline 1.2 Marketing y Calidad & & & & D & & & & & & & \\
\hline 1.1 Ingeniería de Producto & & & & & & & $\mathbf{G}$ & & $\mathbf{I}$ & & \\
\hline \multicolumn{12}{|l|}{ 1. Básico: realiza algunas actividades } \\
\hline SECTORES & \multicolumn{4}{|c|}{ Lácteos } & \multicolumn{4}{|c|}{ Insumos } & \multicolumn{3}{|c|}{ Carnes } \\
\hline
\end{tabular}

\subsection{Implicancias de los niveles de madurez}

Se pueden observar dos grandes grupos de empresas: las que se encuentran en un nivel básico de madurez y las que se encuentran en un nivel intermedio. Esto tiene implicancias para gestionar el PDP con mayor o menor efectividad.

En las organizaciones pertenecientes al primer grupo, el PDP se sustenta en una estrategia que sólo es conocida y manejada por los directivos. Este nivel se caracteriza porque los procesos se mantienen sin sufrir cambios dado que existe una aversión a la innovación para evitar desequilibrios (funcionar sin alterar). Estos aspectos dificultan una gestión sistemática del PDP al estar estrechamente vinculado a la figura del gerente/propietario.

Las empresas del segundo grupo se caracterizan por la aplicación de conocimientos y tecnologías para la mejora del PDP, contando con algún nivel de estandarización en la mayoría de los procesos. Ello permite gestionar el PDP a partir, fundamentalmente, de indicadores de desempeño.

\section{CONCLUSIONES}

El objetivo del presente trabajo ha sido realizar un diagnóstico del nivel de madurez de la Gestión del Proceso de Desarrollo de Productos (PDP) en empresas productoras de alimentos del Gran Santa Fe (Argentina). Se utilizó como referencia la clasificación propuesta por Rozenfeld et al. (2006).

Entre las principales conclusiones se evidencia que solo algunas de las empresas disponen de metodologías y prácticas internalizadas para la Gestión del PDP. Además, existen notorias diferencias en los niveles de detalle que presenta la sistematización de las actividades.

Se observa un grupo de empresas, A, B, C, D y E (empresas medianas y grandes de los sectores lácteo e insumos) que disponen de un modelo de Gestión de PDP definido y que las metodologías y prácticas del PDP se encuentran internalizadas para su aplicación sistemática; mientras que otro grupo, compuesto por I (empresa mediana del sector lácteo), J, K (empresas 
pequeña y mediana del sector insumos) y $\mathrm{F}, \mathrm{G}$ y $\mathrm{H}$ (empresas pequeñas y grande del sector carnes), poseen escasa sistematización en la Gestión del PDP.

Ello permitiría concluir que las diferencias pueden observarse, fundamentalmente, a nivel individual, en general asociadas al tamaño de las organizaciones (a mayor tamaño, mayor nivel de madurez del PDP). Por otro lado, la información indica que, a partir de la comparación entre los diferentes sectores, el sector de carnes el de menor nivel de desarrollo del PDP. Se destaca que el presente trabajo presenta resultados preliminares, y sería necesario el análisis de un número mayor de empresas para poder ser concluyente en este sentido.

La utilización de un modelo referencial auxilia, organiza y direcciona el análisis del PDP. El Modelo de Rozenfeld et al. (2006) resultó de utilidad, dado que permite la comparación de los niveles de madurez del PDP. En relación a la propuesta de los autores, las empresas del segundo grupo deberían comenzar con la aplicación de una serie de actividades (Proyectos de Intervención en la Gestión del PDP) que brinden prácticas y herramientas apropiadas para superar las brechas detectadas con las prácticas del primer grupo, de forma de conseguir mejoras parciales e incrementales en el proceso.

\section{REFERENCIAS}

ABU, N. et al. The pre-development process implementation of product innovation: A Malaysian food and beverage manufacturing SMEs survey. International Journal of Business and Management Science, v. 5, n. 1, p. 39-49, 2012.

AGUIRRE MAYORGA, S. Y CÓRDOBA PINZÓN, N. Diagnóstico de la madurez de los procesos en empresas medianas colombianas, Ing. Univ. Bogotá (Colombia), vol. 12, $\mathrm{n}^{\mathbf{o}} 2$, 2008.

CHRISSIS, M. et al. CMMI: guidelines for processs integration and product improvement. USA: Adiison-Wesley, 2003.

DE GREEF, M., ARCUSIN, L. Y ROSSETTI, G. Tools Description for Product Development Process Management in Food Industries, International Journal of Research in Engineering and Technology, vol. 03, issue 11, 2014.

LERMA KIRCHNER, A. Desarrollo de nuevos productos, una visión integral. Cuarta edición. Querétaro, México: Cengage Learning, 2010.

PENSO, C. Modelo de Referência para o Processo de Desenvolvimento de Produtos na Indústria de Alimentos. Tese de Mestrado, Universidad Federal de Santa Catarina, Florianópolis, Brasil, 2003.

ROSSETTI, G. Y ARCUSIN, L. Diagnóstico del Nivel de Madurez de la Gestión del Proceso de Desarrollo de Productos en Empresas Productoras de Fármacos, Strategy \& Management Business Review, vol. 4, nº 2, 2013.

ROZENFELD, H. et al. Gestão de Desenvolvimento de Produtos: uma referência para a melhoria do processo. São Paulo: Saraiva, 2006.

\section{AGRADECIMIENTOS}

Los autores agradecen la contribución económica de la Agencia Nacional de Promoción Científica y Tecnológica, a través del Fondo para la Investigación Científica y Tecnológica (PICT 2015-N¹629) y de la Universidad Nacional del Litoral (CAI+D 2016 PI 50120150100216LI). 